\title{
Impact Tensile Properties of Notched Titanium Alloy Bolt for Fairing Separation of Launch Vehicle
}

\author{
Hiroto Shimanuki ${ }^{1, *}$, Hiroyuki Yamada ${ }^{2}$, Tsuyoshi $\mathrm{Kami}^{3}$, Yoshihiro Yamasaki ${ }^{4}$, Hiroshi Ikaida $^{4}$, Toru Kamita $^{4}$, Hiroaki \\ Amakawa $^{4}$, Miki Nishimoto ${ }^{4}$ and Hidetoshi Kobayashi ${ }^{5}$ \\ ${ }^{1}$ Graduate Student, Graduate School of Engineering Science, Osaka University, 1-3 Machikaneyama, Toyonaka, Osaka \\ 560-8531, Japan \\ ${ }^{2}$ National Defence Academy, 1-10-20 Hashirimizu, Yokosuka, Kanagawa 239-8686, Japan \\ ${ }^{3}$ Graduate Student, National Defence Academy, 1-10-20 Hashirimizu, Yokosuka, Kanagawa 239-8686, Japan \\ ${ }^{4}$ Japan Aerospace Exploration Agency, 2-1-1 Sengen, Tsukuba, Ibaraki 305-8505, Japan \\ ${ }^{5}$ Graduate School of Engineering Science, Osaka University, 1-3 Machikaneyama, Toyonaka, Osaka 560-8531, Japan
}

\begin{abstract}
The payload fairing in Japan is fixed by a lot of notched bolts. These notched bolts were fractured by axial impact tensile using the explosive devices to separate the fairing. In this case, the stress waves and the oscillations propagate, which may seriously damage the satellites. In this study, the impact deformation and the fracture behavior of notched titanium alloy bolt was investigated using a split Hopkinson pressure bar method. The notched bolt specimen was made of commercial Ti-6Al-4V alloy. The maximum load value was increased with increasing the displacement rate. It can be said that the strain rate dependence of strength for Ti-6Al-4V alloy appeared. From the observation of fracture surface using a scanning electron microscope, compared with the quasi-static test, it was clear that the irregularities of the fractured surface at the impact tensile test became rough. Therefore, it was found that the brittle fracture was mainly observed due to the increase in displacement rate, which may mean that the mode of fracture changes from the transgranular to the intergranular. It was surmised that this change of fracture mode was caused by the high strain rate due to stress concentration of the notched part.
\end{abstract}

\section{Introduction}

The payload fairing is one of the components of the launch vehicle, which has the role of protecting its body and the mounted satellites from the dynamic pressure and aerodynamic heating during the flight to leave the atmosphere.

The payload fairing in Japan launch vehicle is fixed by a lot of notched bolts. When the launch vehicle reached the outer atmosphere, these notched bolts were fractured by axial impact tensile using the explosive devices in order to separate the fairing from the launch vehicle. In this case, the stress waves and the oscillations propagate, which may seriously damage the mounted satellites. In order to successfully complete the mission of the launch vehicle, it is important to mitigate the impact phenomenon against the satellite.

Therefore, the quantitative evaluation of the impact phenomenon in the satellite is required using numerical analysis. However, the current prediction method of that phenomenon is mainly distance attenuation, coupled damping, and branch damping, which is not the promising technique for accuracy. Therefore, the elucidation of fairing separation mechanism and its application to the finite element analysis are required. For that reason, it is necessary to experimentally investigate the impact fracture phenomenon of the notched bolt.

In this study, the impact deformation and the fracture behaviour of the notched titanium alloy bolt for fairing separation of launch vehicle was investigated by using a split Hopkinson pressure bar (SHPB) method [1].

\section{Notched bolt specimen}

In the present study, the Ti-6Al-4V alloy, which is one of commercial titanium alloys, was used. The chemical composition of the Ti-6Al-4V alloy is shown in Table 1. The specimen was prepared by lathe machining based on the shape of the notched bolt used for the actual fairing separation. Figure 1 shows the photograph of the notched titanium alloy bolt specimen. As a result of simple numerical analysis by the explicit method, it was confirmed that the notched part of the specimen was plastically deformed.

Table 1. Chemical composition of Ti-6Al-4V alloy (mass\%).

\begin{tabular}{|c|c|c|c|c|c|c|}
\hline Alloy & $\mathrm{C}$ & $\mathrm{O}$ & $\mathrm{Fe}$ & $\mathrm{Al}$ & $\mathrm{V}$ & $\mathrm{Ti}$ \\
\hline Ti-6Al-4V & 0.01 & 0.15 & 0.15 & 6.28 & 4.1 & Bal. \\
\hline
\end{tabular}

\footnotetext{
E-mail: hiroto.shimanuki@impact.me.es.osaka-u.ac.jp
} 


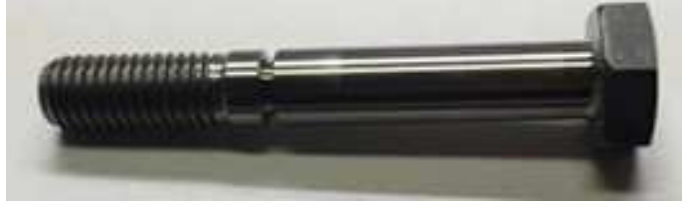

Fig. 1. Photograph of notched titanium alloy bolt specimen.

\section{Experimental}

\subsection{Quasi-static tensile test}

The quasi-static tensile test was conducted using an universal testing machine (Instron, 5500R) at room temperature. The rate of the crosshead was $8.3 \times 10^{-6} \mathrm{~m} / \mathrm{s}$ and $8.3 \times 10^{-5} \mathrm{~m} / \mathrm{s}$. The rate of the crosshead was taken as the displacement rate of the specimen.

\subsection{Impact tensile test by split Hopkinson pressure bar method}

The SHPB method is one of reliable methods among impact deformation tests. This test method can evaluate the impact deformation characteristics by precisely measuring the stress-strain relation of the specimen sandwiched between two elastic round bars by utilizing elastic stress wave propagation. Figure 2 shows a schematic diagram of the apparatus for SHPB type impact tensile test [2].

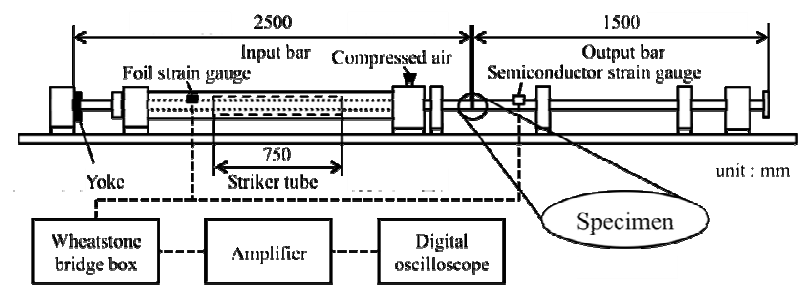

Fig. 2. Apparatus for SHPB type impact tensile test.

A strain pulse (incident wave $\varepsilon_{\mathrm{i}}(\mathrm{t})$ ) propagates through the input bar when a striker tube accelerated by air pressure collides with a yoke the end of the input bar. The strain pulse reached the specimen is reflected to the input bar (reflected wave $\varepsilon_{\mathrm{r}}(\mathrm{t})$ ) and the rest is transmitted through the specimen to the output bar (transmitted wave $\varepsilon_{\mathrm{t}}(\mathrm{t})$ ). These strain pulses were measured with the strain gauges attached on the input and the output bars, respectively. The measured strain pulse was stored in a digital oscilloscope via the Wheatstone bridge circuit and the differential amplifier.

Figure 3 shows an example of strain-time relation in the impact tensile test. It was confirmed that rectangular incident waves are propagated and data could be acquired until fracture of specimen.

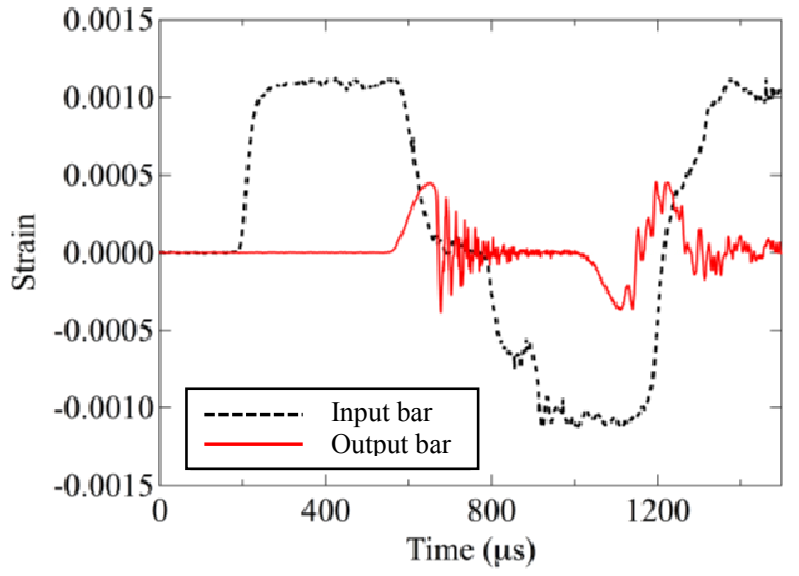

Fig. 3. Strain-time relation of the input and the output bars in the SHPB type impact tensile test.

The load $P$ and displacement $h$ as a function of time are obtained from one-dimensional elastic wave propagation theory as follows:

$$
\begin{gathered}
P(t)=A E \varepsilon_{t}(t) \\
h(t)=2 c_{0} \int_{0}^{t}\left[\varepsilon_{i}(t)-\varepsilon_{t}(t)\right] d t
\end{gathered}
$$

where the Young's modulus, the cross-sectional area, the elastic wave velocity of the input and the output bars are $E, A, c_{0}$.

The displacement rate, $\dot{h}$, is calculated by the time derivative of equation (2). The obtained displacement rate is from approximately $3 \times 10^{0}$ to $6 \times 10^{0} \mathrm{~m} / \mathrm{s}$.

\section{Results of tensile test}

Figs. 4 and 5 show the typical load-displacement relation of the notched Ti-6Al-4V alloy bolt in the quasi-static and the impact tensile tests. These tests results are shown separately because the compliance of the test apparatus is different. In all test conditions, the load-displacement relations showed the elastic deformation, and then the specimen immediately fractured with very little plastic deformation. In this study, we focus on the maximum load value as the characteristic data in load-displacement relations.

Figure 6 shows the relation between the maximum load value and the displacement rate. The maximum load value showed a tendency to increase with increasing displacement rate. In the previous studies, the Ti-6Al-4V alloys are known to have the strain rate dependence of material strength [3-5]. Therefore, it can be said that this dependence also appeared in the notched Ti-6Al-4V alloy bolt.

In order to consider the displacement rate dependence in more detail, the power law approximation was performed on the relation between the maximum load value and the displacement rate. An approximate curve is also shown in Fig. 6, which is expressed as follows:

$$
P_{\max }=C v^{\alpha}
$$


where $P_{\max }$ is the maximum load value, and $v$ is the displacement rate. $C$ is the material constant value, which is unrelated to the displacement rate. $\alpha$ is the increased rate of the maximum load value with increasing displacement rate, which is defined as the rate sensitivity of the notched Ti-6Al-4V alloy bolt.

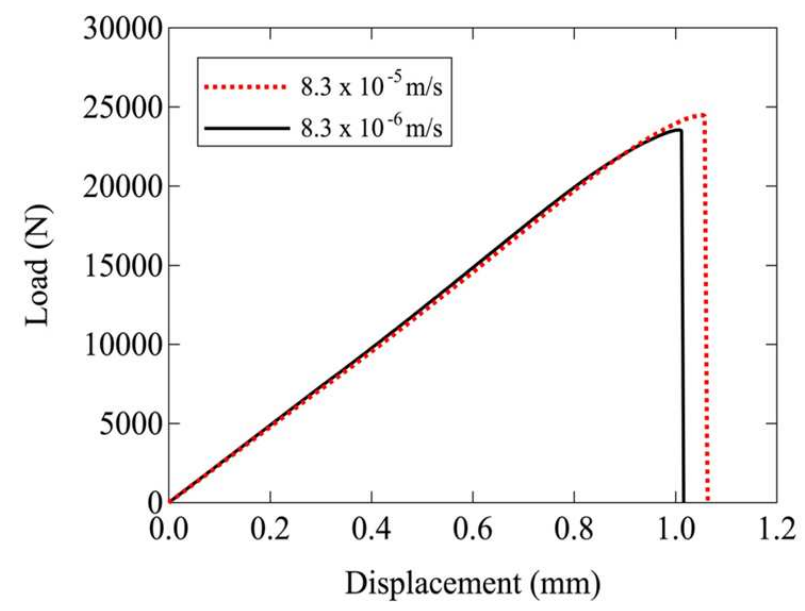

Fig. 4. Load-displacement relation during the quasi-static deformation in the notched Ti-6Al-4V alloy bolt.

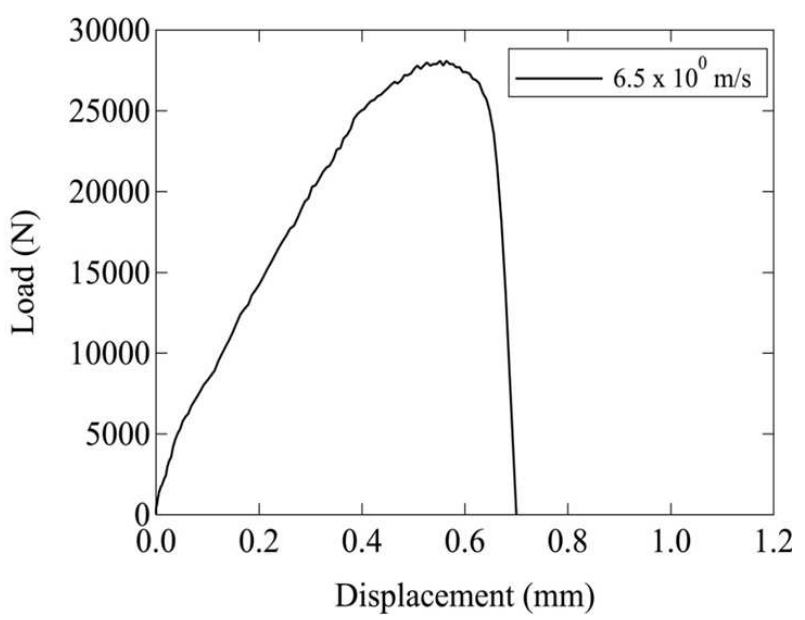

Fig. 5. Load-displacement relation during impact deformation in the notched Ti-6Al-4V alloy bolt.

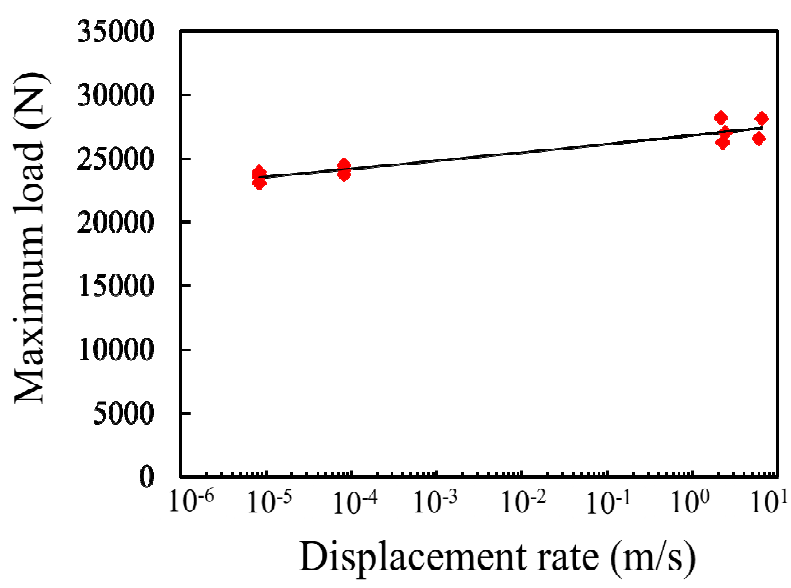

Fig. 6. Maximum load-displacement rate relation in the notched Ti-6Al-4V alloy bolt.
As shown by the solid line in Fig. 6, $C$ and $\alpha$ were obtained as 26524 and 0.014 , respectively. The relation between the maximum load value and the displacement rate of the notched Ti-6Al-4V alloy bolt can be predicted from equation (3).

As with the above experiments, by performing the quasi-static and the impact tensile tests, it is possible to obtain the material constitution equation of the actual notched titanium alloy bolt used for fairing separation of launch vehicle as shown in equation (3). Therefore, the improvement of the accuracy of numerical analysis can be expected.

\section{Results of fracture surface observation}

The fracture surfaces of the quasi-static and the impact tensile tests were observed using a scanning electron microscope (SEM). The results are shown in Figs. 7 and 8.

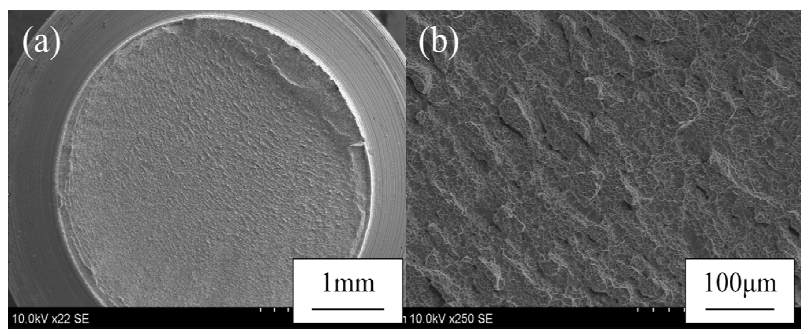

Fig. 7. SEM images of the notched Ti-6Al-4V alloy bolt in the quasi-static tensile test (a) and an enlarged view (b).

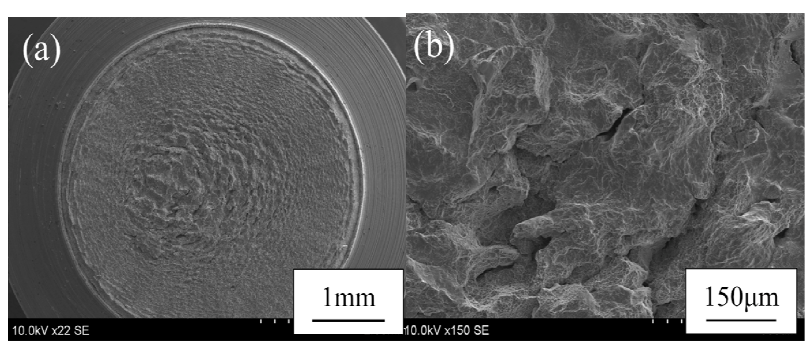

Fig. 8. SEM images of the notched Ti-6Al-4V alloy bolt in the impact tensile test (a) and an enlarged view (b).

In the quasi-static tensile test, the ductile dimples were mainly observed, and the brittle fracture with the irregularities was confirmed in vicinity of the notched part. On the other hand, in the impact tensile test, the brittle fracture was mainly observed in the center. Furthermore the fracture surfaces mixed of the brittle and the ductile were observed in vicinity of the notched part.

Compared with the quasi-static tensile test, it was clear that the irregularities of the fractured surface at the impact tensile test became rough. Therefore, it was found that the brittle fracture was mainly observed due to the increase in displacement rate, which may mean that the mode of fracture changes from the transgranular to the intergranular. It was surmised that this change of fracture mode was caused by the high strain rate due to stress concentration of the notched part. 
There was a difference between the quasi-static and the impact tensile tests on the place where the brittle fracture occurred. The elucidation of this mechanism is a future study.

\section{Summary}

In this study, we tried to elucidate the impact deformation and the fracture behavior of the notched titanium alloy bolt for fairing separation of launch vehicle. The obtained results are shown below:

(1) It was clarified that the notched Ti-6Al-4V alloy bolt indicated the rate dependence of the material strength. The relation between the maximum load value and the displacement rate is expressed as equation (3).

(2) From the observation of fracture surface, it was found that the notched Ti-6Al-4V alloy bolt was mainly observed the brittle fracture due to an increase in displacement rate, which may mean that the mode of fracture changes from the transgranular to the intergranular.

We are grateful to Dr. Tanigaki (Osaka Univ.) for comments and Mr. Kunugi (Undergraduate student in NDA) for assistance with the impact tensile test.

\section{References}

1. H. Kolsky, Proc. Phys. Soc. B, 62, 676 (1949)

2. H. Yamada, M. Tsurudome, N. Miura, K. Horikawa, N. Ogasawara, Mater. Sci. Eng. A, 642, 194 (2015)

3. K. Ogawa, T. Nojima, Material, 371171 (1988) (in Japanese)

4. W.S. Lee, C.F. Lin, Mater. Sci. Eng. A, 241, 48 (1998)

5. S. Seo, O. Min, H. Yang, Int. J. Impact Eng., 31, 735 (2005) 